\title{
VALORAÇÃO ECONÔMICA DO SERVIÇO DE POLINIZAÇÃO NA AGRICULTURA NO CENTRO-OESTE BRASILEIRO (2010-2018)
}

Francis Régis Gonçalves Mendes Barbosa ${ }^{1}$ Maiara de Oliveira Noronha ${ }^{2}$ Carlos Alberto Piacenti ${ }^{3}$

Resumo: O objetivo desta pesquisa foi estimar o Valor Econômico do Serviço de Polinização Animal (VESP) das atividades agrícolas dependentes de polinização na região Centro-Oeste brasileira no período de 2010 a 2018, ano a ano e em valores presentes de 2018, além de discutir as principais causas e implicações da redução de polinizadores e propor estratégias para mitigação do problema. O VESP foi estimado a partir do valor da produção e da razão de dependência de polinização das culturas. O VESP representou aproximadamente $1 / 6$ de todo 0 valor da produção agrícola. As mudanças no uso e cobertura do solo e o uso de agroquímicos foram algumas causas da redução dos polinizadores e a restauração da vegetação nativa e da diversidade biológica algumas estratégias para mitigação do problema.

Palavras-chave: Serviços Ecossistêmicos. Polinizadores. Polinização Agrícola. Valoração Ambiental.

\section{ECONOMIC VALUATION OF THE AGRICULTURAL POLLINATION SERVICE AT BRAZILIAN CENTER-WEST (2010-2018)}

Abstract: The objective of this paper was to estimate the Economic Value of the Animal Pollination Service (VESP) of the agricultural crops dependent on pollination at Brazilian Center-West region from 2010 to 2018 period, year by year and in present values of 2018, in addition to discussing the main causes and implications of pollinator reduction and proposing strategies to mitigate the problem. The VESP was estimated from the value of production and the ratio of crops dependent on pollination. The VESP represented approximately $1 / 6$ of the total value of agricultural production. Changes in land use and cover and the use of agrochemicals were some causes of pollinators reduction and the restoration of native vegetation and biological diversity some strategies to mitigate the problem.

Keywords: Ecosystem Services. Pollinators. Agricultural Pollination. Environmental Valuation.

\section{VALORACIÓN ECONÓMICA DEL SERVICIO DE POLINIZACIÓN EN LA AGRICULTURA DEL CENTRO-OESTE DE BRASIL}

Resumen: El objetivo de esta investigación fue estimar el Valor Económico del Servicio de Polinización Animal (VESP) de las actividades agrícolas dependientes de

\footnotetext{
${ }^{1}$ Universidade Estadual do Oeste do Paraná, Programa de Pós-Graduação em Desenvolvimento Regional e Agronegócio, Toledo-PR, Brasil; Universidade Estadual de Mato Grosso do Sul (UEMS), Departamento de Ciências Econômicas, Ponta Porã-MS, Brasil, francis_barbosa@hotmail.com, https://orcid.org/0000-0003-4462-7668

2 Universidade Estadual do Oeste do Paraná, Programa de Pós-Graduação em Desenvolvimento Regional e Agronegócio, Toledo-PR, Brasil, mai.ol.noronha@gmail.com, https://orcid.org/0000-0002-7525-2996

${ }_{3}^{3}$ Universidade Estadual do Oeste do Paraná, Programa de Pós-Graduação em Desenvolvimento Regional e Agronegócio, Toledo-PR, Brasil, piacenti8@yahoo.com.br, https://orcid.org/0000-00016874-7379
} 
polinización en la región Centro-Oeste de Brasil de 2010 a 2018, año por año y en valores actuales a partir de 2018, así como discutir las principales causas e implicaciones de la reducción de los polinizadores y proponer estrategias para la mitigación del problema. Lo VESP fue estimado a partir del valor de la producción y de la relación de dependencia de polinización de los cultivos. Lo VESP representó aproximadamente $1 / 6$ del valor total de la producción agrícola. Los cambios en el uso y cubierta del suelo y el uso de agroquímicos fueron algunas causas de la reducción de los polinizadores y la restauración de las vegetación nativa y diversidad biológica son algunas estrategias para mitigar el problema.

Palabras clave: Servicios Ecosistémicos. Polinizadores. Polinización Agrícola. Valoración Ambiental.

\section{Introdução}

Serviços Ecossistêmicos (SE) podem ser entendidos como funções provenientes da natureza (biodiversidade e ecossistemas) que melhoram e dão sustentação ao bem-estar humano (DAILY, 1997). No entanto, há inúmeras conceituações de SE construídas sob uma visão antropocêntrica de produtos resultantes dos SE que beneficiam a vida humana, que comprometem o seu uso para a conservação da natureza ou utilização sustentável dos recursos (LAMARQUE; QUÉTIER; LAVOREL, 2011). Há também uma diversidade de classificações de SE baseadas nos tipos de serviço, produtos derivados e escalas aplicadas, porém uma das mais utilizadas os classificam em serviços de provisão, regulação, cultural e de suporte (KINDLER, 2016).

A lista de serviços ecossistêmicos contempla regulação de gases, clima, de distúrbios e de água, oferta e purificação de água, controle de erosão e retenção de sedimentos, formação do solo, ciclagem de nutrientes, tratamento de resíduos, polinização, controle biológico de pragas e doenças, refúgio, produção de alimentos, matérias-primas e lenha, bioquímica, recursos genéticos, recreação, cultura e herança cultural, espiritualidade e religião, estética e inspiração, educação e senso de lugar (pertencimento) (COSTANZA et al., 1997; ALCAMO; BENNETT, 2003).

No rol de serviços ecossistêmicos se encontra o serviço de polinização animal, prestado por organismos selvagens ou de vida livre. A polinização é prestada principalmente por abelhas selvagens, mas também ocorre por borboletas, mariposas, moscas, besouros e vespas, e outros invertebrados, aves e mamíferos como os morcegos, além de espécies de abelhas domesticadas, notadamente a espécie da abelha de mel (Apis melífera) e suas parentes asiáticas, porém em muitas culturas elas não são eficazes ou são parcialmente polinizadoras (GALLAl; VAISSIÈRE, 2009). O grupo de polinizadores predominante e de maior importância econômica é o das abelhas (KLEIN et al., 2007; KREMEN et al., 2007), contudo os 
demais insetos polinizadores respondem por aproximadamente 39\% das visitas às flores das culturas agrícolas e as realizam com maior frequência que as abelhas, sendo também fundamentais para a produção agrícola global (RADER et al., 2016).

Especificamente a polinização realizada por animais contribui diretamente para a produção de alimentos e outras culturas agrícolas de valor comercial, fibras, forragem, produtos florestais madeireiros e não madeireiros (lenha, produtos medicinais e frutos silvestres) e indiretamente na reprodução de plantas selvagens das quais dependem a provisão de outros serviços ambientais e organismos provedores, os serviços de suporte. Estima-se que um terço da produção agrícola mundial dependa da polinização animal ao passo que 60-90\% das espécies vegetais requeiram um polinizador animal (KREMEN et al., 2007). Ainda, cerca de $75 \%$ das principais culturas alimentares mundiais são influenciadas pela polinização animal (variando o grau de dependência da polinização entre as culturas), sendo esse serviço ecossistêmico responsável pelo aumento da produção de 87 dessas culturas, além de medicamentos derivados de plantas (KLEIN et al., 2007; GALLAl; VAISSIÈRE, 2009). Os benefícios agregados da polinização no mundo aumentaram de US\$ 203 bilhões em 1993 para US $\$ 361$ bilhões em 2009 (LAUTENBACH, 2019).

A polinização na agricultura provisionada por abelhas pode ser realizada por diversas espécies, algumas passíveis de gestão e reprodução comerciais como a Apis melifera, outras exclusivamente por espécies selvagens nativas. Há evidências de que ocorre o aumento da polinização por abelhas nativas conforme a proximidade das plantações à habitats naturais, tanto na produção convencional (KREMEN et al., 2004) como na orgânica (KREMEN; WILLIAMS; THORP, 2002), enquanto há redução da variedade de espécies e do contingente de abelhas devido à intensificação agrícola, embora essa constatação não possa ser generalizada para todas as atividades agrícolas, regiões e espécies de abelhas (SAMNEGÅRD et al., 2016).

Ademais, além de beneficiarem diretamente a produção agrícola, as abelhas nativas complementam o serviço prestado pelas abelhas melíferas ao aumentar a eficácia de polinização dessas e ao suprir uma parte da escassez de polinização (WINFREE; GROSS; KREMEN, 2011). O serviço de polinização na agricultura é melhorado significativamente com a diversidade de polinizadores e a abundância de polinizadores selvagens, haja vista a diferença de comportamento forrageiro dos polinizadores (EERAERTS; SMAGGHE; MEEUS, 2020). 
A despeito da eficácia da polinização realizada por abelhas na agricultura, foram registradas reduções no estoque desses insetos nos Estados Unidos desde os anos 1940, agravada nos anos 1990 devido à infestação pelo ácaro Varroa destructor (ELLIS; EVANS; PETTIS, 2010) e em 2006 pelo chamado Transtorno de Colapso das Colônias na qual as perdas significativas não foram associadas a sintomas de pragas/patogenias conhecidas (COX-FOSTER; VANENGELSDORP, 2009). A redução de subsídios aos apicultores em vários países e o desinteresse pela sucessão na atividade (GALLAl; VAISSIÈRE, 2009; ROUBIK, 2018), a intoxicação por inseticidas e agrotóxicos, o aumento da susceptibilidade a doenças causadas pela exposição a esses agroquímicos (PETTIS et al., 2013) e a consequente redução do serviço de polinização (STANLEY et al., 2015), a competição dos polinizadores por ninhos e recursos florais (néctar e pólen) que alteram o ciclo reprodutivo (THOMSON; PAGE, 2020), alterações climáticas, a poluição ambiental e introdução de espécies vegetais invasoras (STODDARD, 2017; LAUTENBACH, 2019) e a hibridização da raça africana melífera (Africanized $A$. melífera) com a melífera europeia (KREMEN; WILLIAMS; THORP, 2002) também contribuíram para a redução de polinizadores.

$\mathrm{Na}$ década de 1960 iniciaram as grandes transformações tecnológicas na agricultura mundial, denominadas de Revolução Verde, as quais contribuíram substancialmente para o aumento da produção de alimentos. Na região CentroOeste brasileira essas transformações se materializaram a partir da década de 1970, por meio de intensa expansão da fronteira agrícola e pelas inovações tecnológicas na agropecuária lideradas pela EMBRAPA, organização fundamental para a criação e adaptação de tecnologias de melhoramento da fertilidade dos solos ácidos do Cerrado, desenvolvimento de novas variedades de grãos e pastos, mecanização e uso de insumos químicos, que incrementaram sobremaneira a produtividade do setor (BARROS, 2010; PEREIRA et al., 2012). No entanto, muitas dessas práticas são prejudiciais à saúde humana, ao meio ambiente e à manutenção da biodiversidade (MOONEY; CROPPER; REID, 2005), além de provocarem acentuadas reduções nas áreas de vegetação natural (WILCOCK; NEILAND, 2002), com impactos negativos esperados sobre os serviços de polinização.

Diante do contexto histórico de expansão da fronteira agrícola e advento das inovações agropecuárias direcionadas, sobretudo, à agricultura intensiva, mecanizada e monocultora na região Centro-Oeste, e o consequente aumento da produção de culturas relativamente dependentes de polinização, tais como algumas 
variedades de soja, girassol e outras presentes na Região, urge saber qual a dimensão dos serviços de polinização animal no Centro-Oeste diante do cenário de redução global de polinizadores, com possíveis reflexos sobre a produção agrícola. O objetivo desta pesquisa é estimar o Valor Econômico do Serviço de Polinização Animal (VESP) nas atividades agrícolas dependentes de polinização na região Centro-Oeste no período de 2010 a 2018, ano a ano e em valores presentes de 2018, além de discutir as principais causas e implicações da redução de polinizadores e propor estratégias para mitigação do problema. A região CentroOeste, em 2018, foi responsável por $27,94 \%$ do valor da produção e $35 \%$ da área plantada de todas as lavouras temporárias e permanentes do Brasil, 15,42\% da área plantada nacional de feijão, 90,05\% da área de girassol, 28,57\% da área de marmelo, $44,55 \%$ da área da soja e $26,75 \%$ da área do tomate, todas culturas dependentes em alguma medida de polinização (IBGE, 2020).

Para tanto, foi utilizada a metodologia proposta Gianinni et al. (2015a) e atualizadas as estimativas para a região Centro-Oeste até o ano de 2018, metodologia construída a partir das contribuições de Klein et al. (2007) e Gallai e Vaissière (2009), apresentadas na seção 2 desta pesquisa. Na seção 3 constam as estimativas do valor econômico da polinização animal na agricultura centro-oestina e as discussões pertinentes e na seção 4 as considerações finais.

\section{Metodologia}

Com base na atualização dos dados propostos por Klein et al. (2007), considerando apenas as atividades agrícolas encontradas no Brasil, foi desenvolvida uma lista das principais culturas agrícolas do Centro-Oeste brasileiro e suas respectivas razões de dependência de polinização mediada por animais, para se chegar às estimativas do valor econômico dos serviços de polinização para cada atividade agrícola (GIANNINI et al., 2015a). Foram consideradas as espécies vegetais de importância econômica, seja para alimentação humana, vestuário, pecuária, biocombustíveis ou outras finalidades comerciais.

As razões de dependência para cada atividade agrícola mundial de importância econômica propostas por Klein et al. (2007) foram adaptadas à realidade brasileira com base num levantamento da literatura nacional sobre polinizadores e culturas agrícolas (GIANNINI et al., 2015b). Foram utilizadas quatro classes de Razões de Dependência de Polinização (RD), essencial, grande, 
modesta e pequena (KLEIN et al., 2007), algumas atualizadas de acordo com as estimativas de dependência de polinização das atividades agrícolas brasileiras (GIANNINI et al., 2015a).

Os níveis médios de dependência da polinização animal por tipo de cultura agrícola foram extraídos de Klein et al. (2007), interpretadas como o percentual de perda de rendimentos comerciais da atividade agrícola na ausência dos serviços de polinização animal. Na polinização classificada como essencial, o valor médio de redução de rendimento da cultura por polinização é situado entre 90\%-100\% $(\mathrm{RD}=0,95)$; na classificada como grande a perda média de rendimento está situada entre 40\%-90\% ( $R D=0,65)$; na classificada como modesta a redução média varia entre 10\%-40\% ( $R D=0,25)$; na classificada como pequena a redução média de rendimento varia entre $0 \%-10 \%(\mathrm{RD}=0,05)$. Por fim, há culturas agrícolas cuja razão de dependência de polinização ainda é desconhecida pela literatura, classificadas então como desconhecida.

Em seguida, de acordo com o método proposto por Gallai e Vaissière (2009), multiplica-se a RD pelo Valor da Produção Anual (VPA) das atividades agrícolas para se obter as estimativas do Valor Econômico dos Serviços de Polinização Animal (VESP) por atividade agrícola e também o valor total anual que compreende o somatório das estimativas das culturas para o ano (GIANNINI et al., 2015a). As estimativas do VESP não contemplarão as culturas agrícolas cujo VPA for indisponível ou a RD desconhecida. Os dados do VPA do período de 2010 a 2018 foram obtidos da Pesquisa Agrícola Municipal (IBGE, 2020a) realizada anualmente pelo Instituto Brasileiro de Geografia e Estatística (IBGE). O cálculo do VESP anual é realizado conforme a Equação (1):

$$
V E S P_{t=} \sum_{j=1}^{62}\left(R D_{j} * V P A_{j}\right)
$$

Onde $\mathrm{RD}_{j}$ é Razão de Dependência de Polinização da atividade agrícola $j$ (em escala decimal), VPA $j$ é o Valor da Produção Anual da atividade agrícola $j$ (em milhões R\$) e VESP $t$ é Valor Econômico dos Serviços de Polinização animal no período $t$ (em milhões $\mathrm{R} \$$ ). Além do VESP, Giannini et al. (2015a) levantaram outros benefícios dos polinizadores às respectivas atividades agrícolas não restritos à frutificação, a exemplo do aumento da qualidade da fruta ou a produção de sementes.

Para a obtenção do VESP do período em análise (2010 a 2018) a valores presentes de 2018 (VEPSP total), será utilizada uma adaptação do método convencional de cálculo do valor presente de séries (ASSAF NETO, 2012), onde no 
lugar da taxa de desconto será utilizada a taxa de inflação anual (em \%) medida pelo Índice Nacional de Preços ao Consumidor Amplo (IPCA), índice de inflação oficial do governo brasileiro e calculado pelo IBGE (IBGE, 2020b). O método de cálculo do VEPSPtotal é apresentado na Equação (2):

$$
\text { VEPSP }_{\text {total }}=\left[\frac{V E S P_{t}}{\left(1+I P C A_{\mathrm{t}}\right)}\right]+\left[\frac{V E S P_{t+1}}{\left(1+I P C A_{\mathrm{t}}\right) *\left(1+I P C A_{\mathrm{t}+1}\right)}\right]+\cdots+\left[\frac{V E S P_{t+8}}{\left(1+I P C A_{\mathrm{t}}\right) * \mathrm{~m} *\left(1+I P C A_{\mathrm{t}+\mathrm{g}}\right)}\right]
$$

Onde VEPSP total é o valor econômico presente dos serviços de polinização animal na agricultura centro-oestina a valores de 2018 (em milhões de R\$) e IPCA é $_{t}$ a taxa de inflação anual (em escala decimal) medida pelo IPCA no período $t$, onde 0 instante $(t=0)$ representa o ano de 2010 e o instante $(t+8)$ o ano de 2018.

Apesar de amplamente difundidas, as abordagens de valoração econômica de serviços ambientais baseadas na valor da produção são alvo das seguintes críticas metodológicas: i) ignoram a possibilidade de adaptações por parte do agricultor durante o ciclo de produção que poderiam reduzir as perdas de produção; ii) não faz distinção entre a contribuição média e a contribuição marginal dos polinizadores (WINFREE; GROSS; KREMEN, 2011). Contudo, a facilidade de sua aplicação e a disponibilidade de dados aliados à fundamentação teórica da qual foi resultante advogam favoravelmente à utilização dessas abordagens na avaliação econômica de serviços ecossistêmicos como o de polinização na agricultura, não somente pela comunidade científica, mas também por governos e stakeholders envolvidos nas atividades agrícolas.

\section{O valor econômico da polinização no Centro-Oeste brasileiro}

A Tabela 1 apresenta as estimativas do Valor Econômico dos Serviços de Polinização Animal (VESP) da agricultura centro-oestina referente a 62 culturas agrícolas. Dessas, 5 culturas $(8,06 \%)$ foram classificadas como essenciais em relação à dependência de polinização (RD) para a produção (cacau, maracujá, melancia, melão e urucum), 9 culturas $(14,51 \%)$ classificadas com grande dependência de polinização (abacate, girassol, goiaba, guaraná, maçã, marmelo, pera, pêssego e tomate) e 9 culturas $(14,51 \%)$ com modesta dependência de polinização (algodão herbáceo, café, castanha de caju, coco-da-baía, fava, figo, laranja, mamona e soja).

Ainda, 9 culturas (14,51\%) foram classificadas com pequena dependência de polinização (amendoim, caqui, dendê, ervilha, feijão, limão, linho, mamão e tangerina), 28 culturas $(45,16 \%)$ em que a polinização não aumenta a produção 
(entre elas abacaxi, algodão herbáceo, cana-de-açúcar, mandioca, milho, trigo e uva) e 2 culturas (3,22\%) em que os efeitos da polinização sobre a produção são desconhecidos (açaí e tungue), conforme classificações propostas por Klein et al., (2007) e Giannini et al., (2015a). 
BARBOSA, NORONHA, PIACENTI

Tạbela 1. Razão de dependência de polinização (RD, em \%), e Valor Econômico dos Serviços de Polinização Animal (VESP, em milhões R\$) das lavouras temporárias e permanentes da região Centro-Oeste (2010-2018)

\begin{tabular}{|c|c|c|c|c|c|c|c|c|c|c|c|c|c|}
\hline \multirow{3}{*}{ Cultura agrícola } & \multirow{2}{*}{\multicolumn{2}{|c|}{$\begin{array}{c}\text { Dependência de } \\
\text { polinizadores na produção }\end{array}$}} & \multirow{3}{*}{$\mathrm{RD}$} & \multicolumn{9}{|c|}{ VESP (milhões R\$) } & \multirow{3}{*}{$\begin{array}{l}\text { Outros benefícios } \\
\text { da polinização, } \\
\text { além da } \\
\text { frutificação } \\
\text { (GIANNINI et al., } \\
\text { 2015a) }\end{array}$} \\
\hline & & & & \multirow[b]{2}{*}{2010} & \multirow[b]{2}{*}{2011} & \multirow[b]{2}{*}{2012} & \multirow[b]{2}{*}{2013} & \multirow[b]{2}{*}{2014} & \multirow[b]{2}{*}{2015} & \multirow[b]{2}{*}{2016} & \multirow[b]{2}{*}{2017} & \multirow[b]{2}{*}{2018} & \\
\hline & $\begin{array}{l}\text { (KLEIN et al., } \\
\text { 2007) }\end{array}$ & $\begin{array}{l}\text { (GIANNINI et } \\
\text { al., 2015a) }\end{array}$ & & & & & & & & & & & \\
\hline Abacate & grande & grande & 0,65 & 1,77 & 0,35 & 4,50 & 3,39 & 2,70 & 5,60 & 3,46 & 6,70 & 5,05 & $\begin{array}{l}\text { aumenta a } \\
\text { produção de } \\
\text { sementes }\end{array}$ \\
\hline Abacaxi & - & não aumenta & 0 & 0 & 0 & 0 & 0 & 0 & 0 & 0 & 0 & 0 & $\begin{array}{c}\text { aumenta a } \\
\text { eficiência } \\
\text { reprodutiva }\end{array}$ \\
\hline Açaí & desconhecida & desconhecida & - & - & - & - & - & - & - & - & - & - & - \\
\hline Algodão arbóreo & modesta & - & 0,25 & 0 & 0 & 0 & 0 & 0 & 0 & 0 & 0 & 0 & - \\
\hline Algodão herbáceo & não aumenta & - & 0 & 0 & 0 & 0 & 0 & 0 & 0 & 0 & 0 & 0 & - \\
\hline Alho & - & não aumenta & 0 & 0 & 0 & 0 & 0 & 0 & 0 & 0 & 0 & 0 & $\begin{array}{c}\text { aumenta a } \\
\text { eficiência } \\
\text { reprodutiva }\end{array}$ \\
\hline Amendoim & baixa & - & 0,05 & 0,53 & 0,24 & 0,22 & 0,23 & 0,29 & 0,17 & 0,28 & 0,44 & 0,44 & - \\
\hline Arroz & não aumenta & - & 0 & 0 & 0 & 0 & 0 & 0 & 0 & 0 & 0 & 0 & - \\
\hline Aveia & não aumenta & - & 0 & 0 & 0 & 0 & 0 & 0 & 0 & 0 & 0 & 0 & - \\
\hline Azeitona & não aumenta & - & 0 & 0 & 0 & 0 & 0 & 0 & 0 & 0 & 0 & 0 & - \\
\hline Banana & - & não aumenta & 0 & 0 & 0 & 0 & 0 & 0 & 0 & 0 & 0 & 0 & aumenta a \\
\hline Batata-doce & - & não aumenta & 0 & 0 & 0 & 0 & 0 & 0 & 0 & 0 & 0 & 0 & eficiência \\
\hline Batata-inglesa & - & não aumenta & 0 & 0 & 0 & 0 & 0 & 0 & 0 & 0 & 0 & 0 & reprodutiva \\
\hline Cacau & essencial & - & 0,95 & 2,69 & 3,19 & 2,15 & 1,85 & 2,20 & 2,54 & 2,64 & 2,69 & 4,12 & - \\
\hline Café & modesta & modesta & 0,25 & 27,43 & 27,76 & 33,92 & 28,97 & 27,42 & 30,69 & 35,15 & 27,80 & 28,48 & $\begin{array}{l}\text { aumenta a } \\
\text { qualidade da } \\
\text { fruta }\end{array}$ \\
\hline
\end{tabular}


BARBOSA, NORONHA, PIACENTI

Tabela 1. Razão de dependência de polinização (RD, em \%), e Valor Econômico dos Serviços de Polinização Animal (VESP, em milhões R\$) das lavouras temporárias e permanentes da região Centro-Oeste (2010-2018)

\begin{tabular}{|c|c|c|c|c|c|c|c|c|c|c|c|c|c|}
\hline \multirow{3}{*}{ Cultura agrícola } & \multirow{2}{*}{\multicolumn{2}{|c|}{$\begin{array}{c}\text { Dependência de } \\
\text { polinizadores na produção }\end{array}$}} & \multirow{3}{*}{$\mathrm{RD}$} & \multicolumn{9}{|c|}{ VESP (milhões R\$) } & \multirow{3}{*}{$\begin{array}{l}\text { Outros benefícios } \\
\text { da polinização, } \\
\text { além da } \\
\text { frutificação } \\
\text { (GIANNINI et al., } \\
2015 a)\end{array}$} \\
\hline & & & & \multirow[b]{2}{*}{2010} & \multirow[b]{2}{*}{2011} & \multirow[b]{2}{*}{2012} & \multirow[b]{2}{*}{2013} & \multirow[b]{2}{*}{2014} & \multirow[b]{2}{*}{2015} & \multirow[b]{2}{*}{2016} & \multirow[b]{2}{*}{2017} & \multirow[b]{2}{*}{2018} & \\
\hline & $\begin{array}{l}\text { (KLEIN et al., } \\
\text { 2007) }\end{array}$ & $\begin{array}{l}\text { (GIANNINI et } \\
\text { al., 2015a) }\end{array}$ & & & & & & & & & & & \\
\hline Cana-de-açúcar & não aumenta & - & 0 & 0 & 0 & 0 & 0 & 0 & 0 & 0 & 0 & 0 & - \\
\hline Caqui & pequena & - & 0,05 & 0 & 0 & 0 & 0 & 0 & 0 & 0 & 0 & 0 & - \\
\hline Castanha de caju & grande & modesta & 0,25 & 0,09 & 0,10 & 0,19 & 0,10 & 0,13 & 0,12 & 0,05 & 0,05 & 0,05 & - \\
\hline Cebola & - & não aumenta & 0 & 0 & 0 & 0 & 0 & 0 & 0 & 0 & 0 & 0 & $\begin{array}{c}\text { aumenta a } \\
\text { produção de } \\
\text { sementes }\end{array}$ \\
\hline Centeio & não aumenta & - & 0 & 0 & 0 & 0 & 0 & 0 & 0 & 0 & 0 & 0 & - \\
\hline Cevada & não aumenta & - & 0 & 0 & 0 & 0 & 0 & 0 & 0 & 0 & 0 & 0 & - \\
\hline Chá-da-índia & - & não aumenta & 0 & 0 & 0 & 0 & 0 & 0 & 0 & 0 & 0 & 0 & - \\
\hline Coco-da-baía & modesta & - & 0,25 & 7,13 & 6,23 & 6,92 & 7,05 & 7,74 & 7,93 & 6,05 & 4,09 & 4,36 & - \\
\hline Dendê & pequena & pequena & 0,05 & 0 & 0 & 0 & 0 & 0 & 0 & 0 & 0 & 0 & - \\
\hline Erva-mate & não aumenta & - & 0 & 0 & 0 & 0 & 0 & 0 & 0 & 0 & 0 & 0 & - \\
\hline Ervilha & baixa & - & 0,05 & 0,16 & 0,04 & 0,02 & 0,00 & 0,06 & 0,18 & 0,08 & 0,01 & 0,11 & - \\
\hline Fava & modesta & - & 0,25 & 0 & 0 & 0 & 0 & 0 & 0 & 0 & 0,89 & 0,71 & - \\
\hline Feijão & baixa & baixa & 0,05 & 46,62 & 44,77 & 69,64 & 69,35 & 48,50 & 62,78 & 123,45 & 81,12 & 62,79 & - \\
\hline Figo & modesta & - & 0,25 & 0,0085 & 0,0013 & 0,0015 & 0,0038 & 0,0103 & 0,0068 & 0 & 0 & 0 & - \\
\hline Fumo & - & não aumenta & 0 & 0 & 0 & 0 & 0 & 0 & 0 & 0 & 0 & 0 & - \\
\hline Girassol & modesta & grande & 0,65 & 23,47 & 24,77 & 51,84 & 49,82 & 71,63 & 72,26 & 43,09 & 57,59 & 89,78 & $\begin{array}{c}\text { aumenta a } \\
\text { produção de } \\
\text { sementes }\end{array}$ \\
\hline Goiaba & - & grande & 0,65 & 11,63 & 12,12 & 19,49 & 17,12 & 12,80 & 15,62 & 11,08 & 16,89 & 13,27 & - \\
\hline Guaraná & - & grande & 0,65 & 0,67 & 0,57 & 0,66 & 0,63 & 2,16 & 1,82 & 1,64 & 1,62 & 0,65 & - \\
\hline
\end{tabular}


BARBOSA, NORONHA, PIACENTI

Tabela 1. Razão de dependência de polinização (RD, em \%), e Valor Econômico dos Serviços de Polinização Animal (VESP, em milhões R\$) das lavouras temporárias e permanentes da região Centro-Oeste (2010-2018)

\begin{tabular}{|c|c|c|c|c|c|c|c|c|c|c|c|c|c|}
\hline \multirow{3}{*}{$\begin{array}{l}\text { Cultura } \\
\text { agrícola }\end{array}$} & \multirow{2}{*}{\multicolumn{2}{|c|}{$\begin{array}{c}\text { Dependência de } \\
\text { polinizadores na produção }\end{array}$}} & \multirow{3}{*}{$\mathrm{RD}$} & \multicolumn{9}{|c|}{ VESP (milhões R\$) } & \multirow{3}{*}{$\begin{array}{l}\text { Outros benefícios } \\
\text { da polinização, } \\
\text { além da } \\
\text { frutificação } \\
\text { (GIANNINI et al., } \\
\text { 2015a) }\end{array}$} \\
\hline & & & & \multirow[b]{2}{*}{2010} & \multirow[b]{2}{*}{2011} & \multirow[b]{2}{*}{2012} & \multirow[b]{2}{*}{2013} & \multirow[b]{2}{*}{2014} & \multirow[b]{2}{*}{2015} & \multirow[b]{2}{*}{2016} & \multirow[b]{2}{*}{2017} & \multirow[b]{2}{*}{2018} & \\
\hline & $\begin{array}{l}\text { (KLEIN et } \\
\text { al., 2007) }\end{array}$ & $\begin{array}{l}\text { (GIANNINI et } \\
\text { al., 2015a) }\end{array}$ & & & & & & & & & & & \\
\hline Juta & - & não aumenta & 0 & 0 & 0 & 0 & 0 & 0 & 0 & 0 & 0 & 0 & - \\
\hline Laranja & pequena & modesta & 0,25 & 16,18 & 15,92 & 15,49 & 16,50 & 18,87 & 19,73 & 25,89 & 26,42 & 43,55 & $\begin{array}{l}\text { aumenta a } \\
\text { qualidade da } \\
\text { fruta }\end{array}$ \\
\hline Limão & pequena & - & 0,05 & 0,499 & 0,503 & 0,488 & 0,529 & 0,751 & 0,768 & 0,770 & 1,204 & 0,703 & - \\
\hline Linho & pequena & - & 0,05 & 0 & 0 & 0 & 0 & 0 & 0 & 0 & 0 & 0 & - \\
\hline Maçã & grande & - & 0,65 & 0 & 0 & 0 & 0 & 0 & 0 & 0 & 0 & 0 & - \\
\hline Malva & - & não aumenta & 0 & 0 & 0 & 0 & 0 & 0 & 0 & 0 & 0 & 0 & - \\
\hline Mamão & pequena & - & 0,05 & 0,341 & 0,322 & 0,258 & 0,225 & 0,476 & 0,387 & 0,622 & 0,510 & 1,111 & - \\
\hline Mamona & - & modesta & 0,25 & 0,008 & 0,045 & 0 & 0 & 0,328 & 0,145 & 0,229 & 0,163 & 0,567 & $\begin{array}{c}\text { Aumenta a } \\
\text { qualidade da } \\
\text { fruta, } \\
\text { quantidade e } \\
\text { qualidade do } \\
\text { óleo } \\
\end{array}$ \\
\hline Mandioca & - & não aumenta & 0 & 0 & 0 & 0 & 0 & 0 & 0 & 0 & 0 & 0 & $\begin{array}{l}\text { aumenta a } \\
\text { eficiência } \\
\text { reprodutiva }\end{array}$ \\
\hline Manga & grande & não aumenta & 0 & 0 & 0 & 0 & 0 & 0 & 0 & 0 & 0 & 0 & - \\
\hline Maracujá & essencial & essencial & 0,95 & 35,47 & 44,50 & 58,24 & 30,84 & 28,11 & 40,65 & 34,36 & 39,98 & 38,01 & - \\
\hline Marmelo & grande & - & 0,65 & 0,072 & 0,105 & 0,058 & 0,000 & 0,000 & 0,235 & 0,169 & 0,133 & 0,144 & - \\
\hline Melancia & essencial & essencial & 0,95 & 114,20 & 148,60 & 152,80 & 145,24 & 148,56 & 168,18 & 182,90 & 195,10 & 155,69 & $\begin{array}{l}\text { aumenta a } \\
\text { qualidade da } \\
\text { fruta }\end{array}$ \\
\hline
\end{tabular}


BARBOSA, NORONHA, PIACENTI

Tabela 1. Razão de dependência de polinização (RD, em \%), e Valor Econômico dos Serviços de Polinização Animal (VESP, em milhões R\$) das lavouras temporárias e permanentes da região Centro-Oeste (2010-2018)

\begin{tabular}{|c|c|c|c|c|c|c|c|c|c|c|c|c|c|}
\hline \multirow{3}{*}{ Cultura agrícola } & \multirow{2}{*}{\multicolumn{2}{|c|}{$\begin{array}{c}\text { Dependência de } \\
\text { polinizadores na produção }\end{array}$}} & \multirow{3}{*}{$\mathrm{RD}$} & \multicolumn{9}{|c|}{ VESP (milhões R\$) } & \multirow{3}{*}{$\begin{array}{c}\text { Outros } \\
\text { benefícios } \\
\text { da } \\
\text { polinização, } \\
\text { além da } \\
\text { frutificação } \\
\text { (GIANNINI } \\
\text { et al., } \\
\text { 2015a) } \\
\end{array}$} \\
\hline & & & & \multirow[b]{2}{*}{2010} & \multirow[b]{2}{*}{2011} & \multirow[b]{2}{*}{2012} & \multirow[b]{2}{*}{2013} & \multirow[b]{2}{*}{2014} & \multirow[b]{2}{*}{2015} & \multirow[b]{2}{*}{2016} & \multirow[b]{2}{*}{2017} & \multirow[b]{2}{*}{2018} & \\
\hline & $\begin{array}{l}\text { (KLEIN et } \\
\text { al., 2007) }\end{array}$ & $\begin{array}{c}\text { (GIANNINI } \\
\text { et al., } \\
2015 a)\end{array}$ & & & & & & & & & & & \\
\hline Melão & essencial & essencial & 0,95 & 0,46 & 0,78 & 1,46 & 4,37 & 5,61 & 4,11 & 6,70 & 3,94 & 3,31 & - \\
\hline Milho & não aumenta & não aumenta & 0 & 0 & 0 & 0 & 0 & 0 & 0 & 0 & 0 & 0 & - \\
\hline $\mathrm{Noz}$ & não aumenta & - & 0 & 0 & 0 & 0 & 0 & 0 & 0 & 0 & 0 & 0 & - \\
\hline Pera & grande & - & 0,65 & 0 & 0 & 0 & 0 & 0 & 0 & 0 & 0 & 0 & - \\
\hline Pêssego & grande & grande & 0,65 & 0 & 0 & 0 & 0 & 0 & 0 & 0 & 0 & 0 & - \\
\hline Pimenta-do-reino & não aumenta & - & 0 & 0 & 0 & 0 & 0 & 0 & 0 & 0 & 0 & 0 & - \\
\hline Rami & - & não aumenta & 0 & 0 & 0 & 0 & 0 & 0 & 0 & 0 & 0 & 0 & - \\
\hline Sisal & - & não aumenta & 0 & 0 & 0 & 0 & 0 & 0 & 0 & 0 & 0 & 0 & - \\
\hline Soja & modesta & modesta & 0,25 & $3.994,78$ & $5.445,57$ & $6.169,52$ & $7.510,88$ & $9.587,34$ & $9.688,19$ & $11.515,44$ & $11.969,97$ & $13.308,22$ & $\begin{array}{c}\text { aumenta a } \\
\text { produção de } \\
\text { sementes }\end{array}$ \\
\hline Tangerina & pequena & - & 0,05 & 0,32 & 0,44 & 0,66 & 0,65 & 0,77 & 0,62 & 0,71 & 1,20 & 1,71 & - \\
\hline Tomate & pequena & grande & 0,65 & 330,94 & 287,45 & 328,60 & 729,69 & 381,13 & 282,21 & 326,34 & 336,95 & 606,59 & $\begin{array}{c}\text { aumenta a } \\
\text { qualidade } \\
\text { da fruta }\end{array}$ \\
\hline Trigo & não aumenta & - & 0 & 0 & 0 & 0 & 0 & 0 & 0 & 0 & 0 & 0 & - \\
\hline Triticale & - & não aumenta & 0 & 0 & 0 & 0 & 0 & 0 & 0 & 0 & 0 & 0 & - \\
\hline Tungue & desconhecida & desconhecida & \begin{tabular}{|l|}
- \\
\end{tabular} & - & - & - & - & - & - & - & - & - & - \\
\hline Urucum & - & essencial & 0,95 & 0,75 & 0,88 & 1,93 & 1,99 & 2,67 & 4,34 & 5,14 & 7,80 & 4,78 & - \\
\hline Uva & não aumenta & não aumenta & 0 & 0 & 0 & 0 & 0 & 0 & 0 & 0 & 0 & 0 & - \\
\hline \multicolumn{4}{|l|}{ Total } & $4.616,23$ & $6.065,27$ & $6.919,06$ & $8.619,45$ & $10.350,25$ & $10.409,31$ & $12.326,24$ & $12.783,25$ & $14.374,20$ & \\
\hline
\end{tabular}


As culturas agrícolas que apresentaram os maiores VESP (em milhões $R \$$ ) durante o período em análise e especificamente em 2018 foram a soja ( $R \$$ 13.308,21 - 92,58\% do VESP), o tomate ( $R \$ 606,59$ - 4,22\%), a melancia ( $R \$$ $155,69$ - 1,08\%), o girassol ( $R \$ 89,79$ - 0,62\%), o feijão ( $R \$ 62,79-0,44 \%)$, a laranja $(R \$ 43,55$ - 0,30\%), o maracujá $(R \$ 38,01$ - 0,26\%) e o café $(R \$ 28,48$ $0,20 \%$ ), respectivamente. Somadas, essas 8 culturas agrícolas foram responsáveis por 99,41\% do VESP em 2010 e por 99,71\% do VESP em 2018, com predominância da cultura da soja que inclusive aumentou sua participação no VESP no período. A participação do VESP no valor da produção total de lavouras temporárias e permanentes do Centro-Oeste evoluiu de 15,31\% em 2010 para 14,98\% em 2018 (IBGE, 2020a), ou seja, a polinização determina aproximadamente 1/6 de todo 0 valor da produção agrícola centro-oestino. Houve um aumento de $211,38 \%$ do VESP no período, evoluindo de $R \$ 4.616,23$ milhões em 2010 para $R \$ 14.374,20$ em 2018.

A soja é a principal atividade agrícola desenvolvida no Centro-Oeste, respondendo em 2018 por 55,58\% do valor da produção agrícola regional e por $56,47 \%$ da área plantada, ao passo que para as outras 7 principais culturas em termos de VESP (somadas) essas participações regionais foram de por 2,94\% e 2,14\% em 2018 (IBGE, 2020a), respectivamente. Tais números explicam a predominância da cultura da soja em termos de VESP e na agricultura centrooestina, situação também observada para o país como um todo (GIANNINI et al., 2015a). As culturas do algodão arbóreo, caqui, dendê e linho, apesar de dependerem em graus variados da polinização animal, não foram produzidas na região Centro-Oeste e, por essa razão, seus respectivos VESP foram nulos. Nulos também foram os VESP de culturas em que a polinização não tem influência sobre a produção (RD nulas) ou que não foram produzidas na região durante o período em análise (a exemplo da maçã, linho, pera e pêssego), conforme a Tabela 1.

Outros benefícios da polinização animal, não restritos à frutificação, foram identificados na literatura (GIANNINI et al., 2015a) em algumas culturas, tais como o aumento da produção de sementes (abacate, cebola, girassol e soja), melhoria da eficiência reprodutiva (abacaxi, alho, banana e mandioca), melhoria da qualidade da fruta (café, laranja, melancia e tomate) e adicionalmente da quantidade e qualidade do óleo extraído, no caso da mamona.

A Tabela 2 apresenta as estimativas do Valor Econômico Presente dos Serviços de Polinização Animal (VEPSP) total (a valores de 2018) e ano a ano. Mesmo com inflação acumulada no período de $69 \%$, foi observado um acréscimo de 
95,09\% do VEPSP de 2010 (R\$ 4.358,64 milhões) para 2018 ( $\mathrm{R} \$$ 8.503,37 milhões) e uma média anual de $R \$ 6.796,72$ milhões, com tendência de crescimento ao longo dos anos, a exceção da transição de 2014 para 2015 na qual ocorreu redução de $9,12 \%$, em parte influenciada pela elevada taxa de inflação registrada em 2015 de 10,67\%. O VEPSP total do período de 2010 a 2018 (VEPSP total) a valores de 2018 foi de $R \$ 61.170,47$ milhões.

Tabela 2. Valor Econômico Presente dos Serviços de Polinização Animal (em milhões R\$),

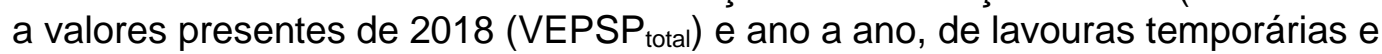
permanentes no Centro-Oeste (2010-2018)

\begin{tabular}{l|ccccccccc}
\hline & 2010 & 2011 & 2012 & 2013 & 2014 & 2015 & 2016 & 2017 & 2018 \\
\hline VESP & $4.616,23$ & $6.065,27$ & $6.919,06$ & $8.619,45$ & $10.350,25$ & $10.409,31$ & $12.326,24$ & $12.783,25$ & $14.374,20$ \\
IPCA & 0,0591 & 0,0650 & 0,0584 & 0,0591 & 0,0641 & 0,1067 & 0,0629 & 0,0295 & 0,0375 \\
VEPSP & $4.358,64$ & $5.377,29$ & $5.795,77$ & $6.817,20$ & $7.692,99$ & $6.990,95$ & $7.788,48$ & $7.845,79$ & $8.503,37$ \\
\hline VEPSP $_{\text {total }}$ & \multicolumn{8}{c}{$61.170,47$} \\
\hline
\end{tabular}

Fonte: Resultados da pesquisa (2020)

Além da soja, as culturas de maior representatividade no Centro-Oeste são o milho, a cana de açúcar e o algodão herbáceo. Essas 4 culturas somadas responderam, em 2018, por 93,81\% do valor da produção agrícola regional e por 95,11\% da área plantada, enquanto em 2010 essas respectivas participações foram de $86,35 \%$ e 92,03\% (IBGE, 2020a). É evidente a preponderância regional de atividades agrícolas monocultoras de produção em larga escala, intensivas em tecnologia e insumos químicos, e a tendência de concentração da produção agrícola em torno dessas atividades.

O predomínio de atividades agrícolas extensivas e monocultoras numa determinada região não está isento de impactos ambientais adversos. A redução da polinização e de outros serviços ecossistêmicos provisionados por animais como o controle de pragas e doenças e dispersão de semente, são resultantes da mudança no uso e cobertura do solo oriundos do avanço da agricultura que geram homogeneização da paisagem, fragmentação e perda de habitats, mudanças microclimáticas e perdas de espécies e populações importantes da fauna (GUTIERREZ-ARELLANO; MULLIGAN, 2018).

Há evidências de que a intensificação da agricultura resultou em perdas significativas na diversidade e abundância total de abelhas nativas, importantes agentes polinizadores devido às flutuações temporais e espaciais das populações de abelhas. $O$ isolamento de recursos florais e de nidificação presentes nas áreas silvestres e o uso de agroquímicos são apontados como os principais motivos da redução dos serviços de polinização prestados por essas abelhas. A restauração da 
vegetação nativa para garantir os recursos florais e de nidificação aliada à redução do uso de agroquímicos são ações que melhorariam os serviços de polinização na agricultura intensiva, além de beneficiar as plantas silvestres, conservando a biodiversidade em toda a paisagem agro-natural (KREMEN; WILLIAMS; THORP, 2002).

A presença e quantidade de habitats seminaturais reduz a sensibilidade dos polinizadores a alterações das condições de temperatura, ao passo que essa sensibilidade aumenta nas áreas de agricultura intensiva (SCHWEIGER et al., 2019). Nesse sentido, alterações genéticas das sementes dos cultivares agrícolas podem aumentar a resistência dessas culturas ao calor e do déficit hídrico, bem como facilitar a polinização (STODDARD, 2017).

Uma estratégia de gestão agrícola para mitigar o declínio de populações de abelhas polinizadoras selvagens é a gestão de faixas florais silvestres. Foram identificadas espécies de flores silvestres capazes de fornecer alimento a uma grande gama de comunidades de abelhas silvestres ao longo do tempo, enquanto outras espécies de flores serviam de forragem somente para espécies específicas de abelhas. Selecionar, plantar e gerir as faixas de flores de espécies silvestres que sustentam uma grande diversidade de abelhas nativas, e em menor proporção as espécies de flores menos visitadas por polinizadores, constituem recomendações práticas para a conservação do serviço de polinização prestado pelas abelhas nativas (BURKLE; DELPHIA; O'NEILL, 2020). Além da gestão paisagística, ações de curto prazo que envolvam o aumento ou redução de polinizadores domesticados (a exemplo das abelhas melíferas) e mudanças no uso de agroquímicos podem aumentar a efetividade da polinização e o rendimento das culturas agrícolas dependentes desse serviço ecossistêmico (GARIBALDI et al., 2020).

Apesar de ameaçadas pela mudança no uso e cobertura do solo oriundos das atividades antrópicas, entre elas a expansão da agricultura monocultora, há evidências de que a maioria dos serviços de polinização nas culturas agrícolas realizado por abelhas é prestado por um número reduzido de espécies em diferentes regiões do mundo, capazes de persistir sob a expansão agrícola e mantidas por simples medidas de conservação. Nesse sentido, a conservação da diversidade biológica das abelhas deve ser motivada não só pelos benefícios imediatos dos serviços ecossistêmicos, mas pela riqueza total dos argumentos a favor da conservação (KLEIJN et al., 2015). 
Por outro lado, os insetos polinizadores não-abelhas, tais como moscas, vespas, besouros e borboletas, são polinizadores eficientes para a agricultura global (fornecem aproximadamente $39 \%$ das visitas florais na agricultura), possuem menor dependência de porções de habitat natural ou seminatural na paisagem circundante às culturas agrícolas, sendo menos sensíveis a alterações do uso do solo, além de realizarem a polinização de forma independente das abelhas. Os insetos polinizadores não-abelhas fornecem um serviço valioso à agricultura e podem compensar o declínio da polinização de abelhas observado na agricultura mundial (RADER et al., 2016).

A quantificação dos benefícios da polinização se constitui um importante incentivo para a conservação de habitats naturais e corredores verdes em áreas agrícolas. Numa análise custo-benefício, deve-se considerar os benefícios sociais, a estética paisagística, a beleza cênica e outros serviços proporcionados por essas áreas, e não somente os custos em termos de terras improdutivas (LAUTENBACH, 2019).

A diversidade biológica pode melhorar a prestação de serviços ecossistêmicos, inclusive os de polinização, aumentando o nível de prestação desses serviços e os estabilizando no tempo (dia ou estações do ano) e espaço (morfologia das flores e plantas). As diferenças no comportamento forrageiro dos insetos polinizadores são uma das razões pelas quais a diversidade e abundância de polinizadores aumenta a polinização das culturas agrícolas (EERAERTS; SMAGGHE; MEEUS, 2020). É previsto pela teoria ecológica que quando um serviço ecossistêmico é fornecido por muitas espécies (diversidade biológica), ele detém uma série de mecanismos estabilizadores de sua provisão (WINFREE; KREMEN, 2009).

Adicionalmente, foi constatado que mudanças na cobertura e uso do solo também afetam outros serviços ecossistêmicos como o de provisão hídrica (GENG et al., 2015), alertando para a necessidade de uma visão holística dos impactos da mudança do uso do solo, inclusive de sua relação com a gestão de recursos hídricos e outros serviços ecossistêmicos, na proposição de políticas para restaurar, conservar e gerir os ecossistemas (CHAUDHARY et al., 2016).

Em regiões altamente dependentes de polinização nos países de baixa renda, a conservação da biodiversidade e dos serviços de polinização é uma questão de segurança alimentar e justiça social, haja vista que a renda e outros meios de sobrevivência de um contingente significativo de agricultores familiares advêm dos 
serviços ecossistêmicos. A valoração monetária dos serviços de polinização fornece uma estimativa dos gastos que poderiam ser executados para proteger os polinizadores nessas regiões. Além da redução do uso de agroquímicos, o aumento de práticas agrícolas ecológicas como a agricultura orgânica e o controle biológico de pragas, a conservação de habitats florestais remanescentes e o fortalecimento de sistemas agrícolas diversificados, a exemplo do sistema agroflorestal, são práticas capazes de proteger as populações de polinizadores (LAUTENBACH, 2019).

Do ponto de vista científico, conciliar a preservação ambiental e a prestação de serviços ecossistêmicos nos agroecossistemas exige infraestruturas transdisciplinares e coordenadas de pesquisa capazes de disponibilizar gratuitamente dados históricos sobre ocorrência de espécies, interações e características dos polinizadores, consolidar protocolos padronizados e coordenados de coleta de dados de longo prazo em áreas naturais e agrícolas e desenvolver infraestruturas de pesquisa multiatores (cientistas, gestores, membros do público, agricultores e disciplinas curriculares) e descentralizadas que permitam a integração de abordagens econômicas, sociais e ecológicas na agricultura (BARTOMEUS; DICKS, 2019).

Em termos de ações governamentais, os planejadores do uso de terras brasileiras precisam estabelecer prioridades conservacionistas da paisagem agrícola, a fim de preservar a agrobiodiversidade e o elo entre a natureza e a agricultura, utilizando de forma racional os serviços ecossistêmicos na agricultura, com destaque para os serviços de polinização (ROCHA, 2012).

Uma das ações nesse sentido é a regulamentação prevista pelo Código Florestal Brasileiro de 1965 e suas atualizações. A regulamentação prevê, dentre outras prerrogativas, a obrigatoriedade, nas propriedades rurais, da composição de: i) Área de Preservação Permanente (APP), área protegida, coberta ou não por vegetação nativa, instituída para preservar os recursos hídricos, a paisagem, a estabilidade geológica e a biodiversidade, proteger o solo e assegurar o bem-estar humano; ii) Reserva Legal, área interior à propriedade rural com a finalidade de assegurar o uso econômico sustentável dos recursos naturais, auxiliar os processos ecológicos, conservar a biodiversidade e proteger a fauna silvestre e flora nativa (BRASIL, 2012). Os produtores podem aproveitar essas áreas exigidas legalmente para fazer a gestão dos habitats naturais, recursos florais e de nidificação em prol da preservação dos polinizadores e demais serviços ecossistêmicos. 


\section{Considerações finais}

A partir da metodologia proposta Gianinni et al. (2015a), foi estimado o Valor Econômico dos Serviço Polinização Animal (VESP) das atividades agrícolas do Centro-Oeste, no período de 2010 a 2018, das produções que dependeram em alguma medida da polinização animal. O VESP evoluiu de $\mathrm{R} \$ 4.616,23$ milhões em 2010 para $\mathrm{R} \$ 14.374,20$ em 2018, aumento de $211,38 \%$ no período, advinda da soja a principal contribuição para esse valor (92,58\% do VESP em 2018), seguida pelas produções de tomate, melancia, girassol, feijão, laranja, maracujá e café, que somadas responderam por 99,41\% em 2010 e 99,71\% do VESP em 2018. No período o VESP foi responsável por aproximadamente $1 / 6$ de todo o valor da produção agrícola centro-oestino. Em termos de valores presentes, o VEPSP aumentou 95,09\% de 2010 para 2018 e contabilizou $R \$$ 61.170,47 milhões no período a valores de 2018.

Na região Centro-Oeste há uma concentração da produção agrícola em torno das atividades de soja, milho, cana de açúcar e algodão herbáceo, que em 2018 responderam por $93,81 \%$ do valor da produção e $95,11 \%$ da área plantada agrícola regional, caracterizadas pela monocultura e intensivas em tecnologia e insumos químicos. Ocorre que as mudanças no uso e cobertura do solo oriundas do avanço desse sistema agrícola são apontadas pela literatura como algumas das principais causas da redução de polinizadores e de outros serviços ecossistêmicos provisionados por animais, sejam eles abelhas, demais insetos ou outros polinizadores.

Outras razões para a redução global de polinizadores na agricultura perpassam pelo uso excessivo de agroquímicos, fragmentação e perda de habitats naturais necessários para a sobrevivência de diversas espécies de polinizadores, mudanças climáticas, a poluição ambiental, introdução de espécies invasoras e as perdas de espécies e populações.

Evidencia-se o trade-off entre o avanço da agricultura intensiva e a produção de alimentos de um lado, e do outro os impactos adversos sobre os ecossistemas e a biodiversidade e a consequente redução dos serviços de polinização, essenciais para a melhoria da produtividade, quantidade e qualidade da produção de diversas culturas. Por outro lado, a redução de polinizadores reduz a renda e os meios de sobrevivência de um contingente global de pequenos produtores rurais, altamente dependentes dos serviços ecossistêmicos, especialmente nos países de baixa renda, constituindo-se uma ameaça à segurança alimentar e à justiça social. 
Diversas ações podem ser realizadas com o intuito de mitigar o problema da redução de polinizadores na agricultura. A restauração da vegetação nativa (inclusive das espécies de flores silvestres) e da diversidade biológica, a redução e administração técnica do uso de agroquímicos, a previsão de orçamento direcionado para a proteção dos polinizadores definido pela valoração monetária dos serviços de polinização, o desenvolvimento de sistemas de produção agrícola sustentáveis como a agricultura orgânica e o sistema agroflorestal, controle biológico de pragas, criação de infraestruturas transdisciplinares e coordenadas de pesquisa pautadas pela visão holística dos impactos da mudança do uso do solo sobre os serviços ecossistêmicos e pela integração das abordagens econômicas, sociais e ecológicas na agricultura, estabelecimento de instituições governamentais conservacionistas na agricultura, a exemplo das exigências de Área de Preservação Permanente e de Reserva Legal nas propriedades rurais previstas pelo Código Florestal Brasileiro e suas atualizações, além de inúmeras outras estratégias não contempladas por esta pesquisa, são caminhos possíveis e exequíveis a se perseguir em busca de novos modelos sustentáveis de agricultura ou no aperfeiçoamento dos já existentes.

Por fim, cabe salientar as limitações metodológicas na estimação do VESP e que esse valor está aquém do efetivo valor dos serviços de polinização na agricultura, haja vista que tais serviços beneficiam toda uma flora silvestre que provisiona as condições de sobrevivência aos polinizadores e outros animais, além de o efeito da polinização em algumas atividades agrícolas ainda ser desconhecido. Fica a sugestão de trabalhos futuros que aperfeiçoem metodologicamente a estimação do valor econômico dos serviços de polinização na agricultura.

\section{REFERÊNCIAS}

ALCAMO, J.; BENNETT, E. M. Ecosystems and human well-being: a framework for assessment. Washington, D.C.: Island Press, 2003.

ASSAF NETO, A. Matemática financeira e suas aplicações. 12. ed. São Paulo: Atlas, 2012.

BARROS, G. S. A. de C. Política agrícola no Brasil: subsídios e investimentos. In: GASQUES, J. G.; VIEIRA FILHO, J. E.; NAVARRO, Z. (Eds.). A agricultura brasileira: desempenho, desafios e perspectivas. Brasília: IPEA, 2010.

BARTOMEUS, I.; DICKS, L. V. The need for coordinated transdisciplinary research infrastructures for pollinator conservation and crop pollination resilience. Environmental Research Letters, v. 14, n. 4, p. 1-9, 2019.

BRASIL. Lei no 12.651, de 25 de maio de 2012. Brasília, DF: Presidência da República, 
2012. Disponpivel em: <http://www.planalto.gov.br/ccivil_03/_ato20112014/2012/lei/l12651.htm>. Acesso em: 20 mar. 2020.

BURKLE, L. A.; DELPHIA, C. M.; O'NEILL, K. M. Redundancy in wildflower strip species helps support spatiotemporal variation in wild bee communities on diversified farms. Basic and Applied Ecology, v. 44, p. 1-13, 2020.

CHAUDHARY, S.; CHETTRI, N.; UDDIN, K.; KHATRI, T. B.; DHAKAL, M.; BAJRACHARYA, B.; NING, W. Implications of land cover change on ecosystems services and people's dependency: A case study from the Koshi Tappu Wildlife Reserve, Nepal. Ecological Complexity, v. 28, p. 200-211, 2016.

COSTANZA, R.; DE GROOT, R.; FARBERLL, S.; GRASSOT, M.; HANNON, B.; LIMBURG, K.; NAEEM, S.; O'NEILL, R. V.; PARUELO, J.; RASKIN, R. G.; SUTTONLLLL, P. The value of the world's ecosystem services and natural capital. Nature, n. 387, p. 253-260, 1997.

COX-FOSTER, D.; VANENGELSDORP, D. Saving the HONEYBEE. Scientific American, v. 300, n. 4, p. 40-47, 2009.

DAILY, G. C. Nature's services: societal dependence on natural ecosystems. Washington, DC: Island Press, 1997.

EERAERTS, M.; SMAGGHE, G.; MEEUS, I. Bumble bee abundance and richness improves honey bee pollination behaviour in sweet cherry. Basic and Applied Ecology, v. 43, p. 2733, 2020.

ELLIS, J. D.; EVANS, J. D.; PETTIS, J. Colony losses, managed colony population decline, and Colony Collapse Disorder in the United States. Journal of Apicultural Research, v. 49, n. 1, p. 134-136, 2010.

GALLAI, N.; VAISSIÈRE, B. Guidelines for the economic valuation of pollination services at a national scale. Roma: FAO, 2009.

GARIBALDI, L. A.; SÁEZ, A.; AIZEN, M. A.; FIJEN, T.; BARTOMEUS, I. Crop pollination management needs flower-visitor monitoring and target values. Journal of Applied Ecology, v. 57, n. 4, p. 664-671, 2020.

GENG, X.; WANG, X.; YAN, H.; ZHANG, Q.; JIN, G. Land use/land cover change induced impacts on water supply service in the upper reach of heihe River Basin. Sustainability, v. 7, n. 1, p. 366-383, 2015.

GIANNINI, T. C.; CORDEIRO, G. D.; FREITAS, B. M.; SARAIVA, A. M.; IMPERATRIZFONSECA, V. L. The dependence of crops for pollinators and the economic value of pollination in Brazil. Journal of Economic Entomology, v. 108, n. 3, p. 849-857, 2015a.

GIANNINI, T. C.; BOFF, S.; CORDEIRO, G. D.; CARTOLANO, E. A.; VEIGA, A. K. IMPERATRIZ-FONSECA, V. L.; SARAIVA, A. M. Crop pollinators in Brazil: a review of reported interactions. Apidologie, n. 46, p. 209-223, 2015b.

GUTIERREZ-ARELLANO, C.; MULLIGAN, M. A review of regulation ecosystem services and disservices from faunal populations and potential impacts of agriculturalisation on their provision, globally. Nature Conservation, n. 30, p. 1-39, 2018.

INSTITUTO BRASILEIRO DE GEOGRAFIA E ESTATÍSTICA (IBGE). Índice Nacional de Preços ao Consumidor Amplo (IPCA). 2020. Disponível em:

<https://www.ibge.gov.br/explica/inflacao.php>. Acesso em: 10 mar. 2020. 
INSTITUTO BRASILEIRO DE GEOGRAFIA E ESTATÍSTICA (IBGE). Produção Agrícola Municipal (PAM). 2020. Disponível em: <http://www.sidra.ibge.gov.br>. Acesso em: 10 mar. 2020.

KINDLER, E. A comparison of the concepts: Ecosystem services and forest functions to improve interdisciplinary exchange. Forest Policy and Economics, v. 67, p. 52-59, 2016.

KLEIJN, D.; WINFREE, R.; BARTOMEUS, I.; CARVALHEIRO, L. G.; HENRY, M.; ISAACS, R.; KLEIN, A. M.; KREMEN, C.; M'GONIGLE, L. K.; RADER, R.; RICKETTS, T. H.; WILLIAMS, N. M.; ADAMSON, N. L.; ASCHER, J. S.; BÁLDI, A.; BATÁRY, P.; BENJAMIN, F.; BIESMEIJER, J. C.; BLITZER, E. J.; BOMMARCO, R.; BRAND, M. R.; BRETAGNOLLE, V.; BUTTON, L.; CARIVEAU, D. P.; CHIFFLET, R.; COLVILLE, J. F.; DANFORTH, B. N.; ELLE, E.; GARRATT, M. P. D.; HERZOG, F.; HOLZSCHUH, A.; HOWLETT, B. G.; JAUKER, F.; JHA, S.; KNOP, E.; KREWENKA, K. M.; LE FÉON, V.; MANDELIK, Y.; MAY, E. A.; PARK, M. G. Delivery of crop pollination services is an insufficient argument for wild pollinator conservation. Nature Communications, v. 6, n. 7414, p. 1-8, 2015.

KLEIN, A. M. VAISSIÈRE, B. E.; CANE, J. H.; STEFFAN-DEWENTER, I.; CUNNINGHAM, S. A.; KREMEN, C.; TSCHARNTKE, T. Importance of pollinators in changing landscapes for world crops. Proceedings of the Royal Society B: Biological Sciences, v. 274, n. 1608, p. 303-313, 2007.

KREMEN, C.; WILLIAMS, N. M.; BUGG, R. L.; FAY, J. P.; THORP, R. W. The area requirements of an ecosystem service: Crop pollination by native bee communities in California. Ecology Letters, v. 7, n. 11, p. 1109-1119, 2004.

KREMEN, C.; WILLIAMS, N. M.; AIZEN, M. A.; GEMMILL-HERREN, B.; LEBUHN, G.; MINCKLEY, R.; PACKER, L.; POTTS, S. G.; ROULSTON, T.; STEFFAN-DEWENTER, I.; VÁZQUEZ, D. P.; WINFREE, R.; ADAMS, L.; CRONE, E. E.; GREENLEAF, S. S.; KEITT, T. H.; KLEIN, A. M.; REGETZ, J.; RICKETTS, T. H. Pollination and other ecosystem services produced by mobile organisms: A conceptual framework for the effects of land-use change. Ecology Letters, v. 10, n. 4, p. 299-314, 2007.

KREMEN, C.; WILLIAMS, N. M.; THORP, R. W. Crop pollination from native bees at risk from agricultural intensification. PNAS, v. 99, n. 26, p. 16812-16816, 2002.

LAMARQUE, P.; QUÉTIER, F.; LAVOREL, S. The diversity of the ecosystem services concept and its implications for their assessment and management. Comptes Rendus Biologies, v. 334, n. 5-6, p. 441-449, 2011.

LAUTENBACH, S. Provisioning ecosystem services at risk: pollination benefits and pollination dependency of cropping systems at the global scale. In: SCHRÖTER, M. et al. (Eds.). Atlas of Ecosystem Services. [s.I.]: Springer, Cham, 2019. p. 97-104.

MOONEY, H.; CROPPER, A.; REID, W. Confronting the human dilemma. Nature, v. 434, n. 31, p. 561-562, 2005.

PEREIRA, P. A. A.; MARTHA JÚNIOR, G. B.; SANTANA, C. A. M.; ALVES, E. The development of Brazilian agriculture: future technological challenges and opportunities. Agriculture \& Food Security, v. 1, n. 4, p. 1-12, 2012.

PETTIS, J. S.; LICHTENBERG, E. M.; ANDREE, M.; STITZINGER, J.; ROSE, R.; VANENGELSDORP, D. Crop pollination exposes honey bees to pesticides which alters their susceptibility to the gut pathogen nosema ceranae. Plos One, v. 8, n. 7, p. e70182, 2013.

RADER, R.; BARTOMEUS, I.; GARIBALDI, L. A.; GARRATT, M. P. D.; HOWLETT, B. G.; WINFREE, R.; CUNNINGHAM, S. A.; MAYFIELD, M. M.; ARTHUR, A. D.; ANDERSSON, G. 
K. S.; BOMMARCO, R.; BRITTAIN, C.; CARVALHEIRO, L. G.; CHACOFF, N. P.; ENTLING, M. H.; FOULLY, B.; FREITAS, B. M.; GEMMILL-HERREN, B.; GHAZOUL, J.; GRIFFIN, S. R.; GROSS, C. L.; HERBERTSSON, L.; HERZOG, F.; HIPÓLITO, J.; JAGGAR, S.; JAUKER, F.; KLEIN, A. M.; KLEIJN, D.; KRISHNAN, S.; LEMOS, C. Q.; LINDSTRÖM, S. A. M.; MANDELIK, Y.; MONTEIRO, V. M.; NELSON, W.; NILSSON, L.; PATTEMORE, D. E.; PEREIRA, O. N.; PISANTY, G.; POTTS, S. G.; SHEFFIELD, C. S.; SCHEPER, J.; SCHÜEPP, C.; SMITH, H. G.; STANLEY, D. A.; STOUT LL, J. C.; TAKI, H.; VERGARA, C. H.; VIANA, B. F.; WOYCIECHOWSKI, M.; REEMER, M.; RUNDLÖF, M. Non-bee insects are important contributors to global crop pollination. PNAS, v. 113, n. 1, p. 146-151, 2016.

ROCHA, M. C. L. S. A. Efeitos dos agrotóxicos sobre as abelhas silvestres no Brasil: proposta metodológica de acompanhamento. 1. ed. Brasília: IBAMA, 2012.

ROUBIK, D. W. Lessons learned over the past 20 years. In: ROUBIK, D. W. (Ed.). The pollination of cultivated plants: a compendium for practitioners. $2^{\circ}$ ed. Rome: FAO, 2018. p. 1-19.

SAMNEGÅRD, U.; HAMBÄCK, P. A.; LEMESSA, D.; NEMOMISSA, S.; HYLANDER, K. A heterogeneous landscape does not guarantee high crop pollination. Proceedings of the Royal Society B: Biological Sciences, v. 283, n. 1838, p. 1-9, 2016.

SCHWEIGER, O.; FRANZÉN, M.; FRENZEL, M.; GALPERN, P.; KERR, J.; PAPANIKOLAOU, A.; RASMONT, P. Minimising risks of global change by enhancing resilience of pollinators in agricultural systems. In: SCHRÖTER, M.; BONN, A.; KLOTZ, S.; SEPPELT, R.; BAESSLER, C. (Eds.). Atlas of Ecosystem Services. [s.I.] : Springer, Cham, 2019. p. 105-111.

STANLEY, D. A.; GARRATT, M. P. D.; WICKENS, J. B.; WICKENS, V. J.; POTTS, S. G.; RAINE, N. E. Neonicotinoid pesticide exposure impairs crop pollination services provided by bumblebees. Nature, v. 528, p. 548-550, 2015.

STODDARD, F. L. Climate change can affect crop pollination in unexpected ways. Journal of Experimental Botany, v. 68, n. 8, p. 1819-1821, 2017.

THOMSON, D. M.; PAGE, M. L. The importance of competition between insect pollinators in the Anthropocene. Current Opinion in Insect Science, v. 38, p. 55-62, 2020.

WILCOCK, C.; NEILAND, R. Pollination failure in plants: why it happens and when it matters. Trends in Plant Sciense, v. 7, n. 6, p. 270-277, 2002.

WINFREE, R.; GROSS, B. J.; KREMEN, C. Valuing pollination services to agriculture. Ecological Economics, v. 71, n. 15, p. 80-88, 2011.

WINFREE, R.; KREMEN, C. Are ecosystem services stabilized by differences among species? A test using crop pollination. Proceedings of the Royal Society B: Biological Sciences, v. 276, n. 1655, p. 229-237, 2009.

\section{NOTAS DE AUTOR}

\section{CONTRIBUIÇÃO DE AUTORIA}

Francis Régis Gonçalves Mendes Barbosa - Concepção. Coleta de dados; Análise de dados; Elaboração do manuscrito, Participação ativa da discussão dos resultados; Revisão e aprovação da versão final do trabalho Maiara de Oliveira Noronha - Concepção e elaboração do manuscrito. Coleta de dados; Participação ativa da discussão dos resultados; Revisão e aprovação da versão final do trabalho.

Carlos Alberto Piacenti - Participação ativa da discussão dos resultados; Revisão e aprovação da versão final do trabalho. 


\section{FINANCIAMENTO}

Não se aplica.

\section{CONSENTIMENTO DE USO DE IMAGEM}

Não se aplica

\section{APROVAÇÃO DE COMITÊ DE ÉTICA EM PESQUISA}

Não se aplica.

\section{CONFLITO DE INTERESSES}

Declaramos não haver conflitos de interesses financeiros, pessoais, entre possíveis revisores e editores e possíveis vieses temáticos.

\section{LICENCA DE USO}

Este artigo está licenciado sob a Licença Creative Commons CC-BY-NC. Com essa licença você pode compartilhar, adaptar, criar para qualquer fim, sem uso comercial e desde que atribua a autoria da obra.

\section{HISTÓRICO}

Recebido em: 28-04-2020

Aprovado em: 18-12-2020 